\title{
Gestión de cuidado de las personas con úlcera venosa: una revisión integrativa
}

\author{
Manejo do cuidado de pessoas com úlcera varicosa: \\ uma revisão integrativa
}

\section{User care management with venous ulcer: an integrative review}

\author{
Juliana da Costa Silva \\ Graduação em Enfermagem - Bacharelado e Licenciatura pela Universidade Federal Fluminense. Mestre em Ciências do Cuida- \\ do em Saúde. Escola de Enfermagem Aurora de Afonso Costa - EEAAC Universidade Federal Fluminense - UFF. Brasil. \\ Cómo citar este artículo en edición digital: Silva, J.C. (2016). Gestión de cuidado de las personas con úlcera venosa: una \\ revisión integrativa. Cultura de los Cuidados (Edición digital), 20( 46). \\ Disponible en: < http://dx.doi.org/10.14198/cuid.2016.46.15> \\ Correspondencia: Escola de Enfermagem Aurora de Afonso Costa - EEAAC Universidade Federal Fluminense - UFF. Brasil \\ Correo electrónico: ju9costa@gmail.com \\ Recibido: 02/08/2015; Aceptado: 24/05/2016
}

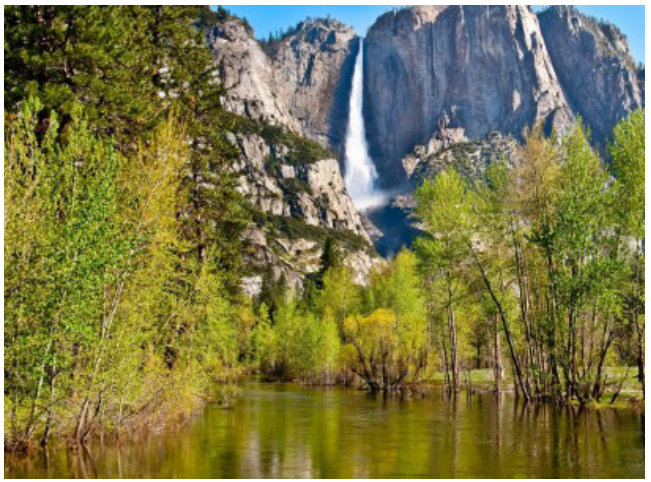

\section{ABSTRACT}

The client with Ulcus Cruris requires proper care, in order to restore health and return to daily activities. The objectives of this study are: to identify the habits of life of the person with varicose ulcer and characterize the forms of care carried out by the person with varicose ulcer. Was conducted an online survey of articles published in the period between 2009 and 2014 who addressed the theme to were searched using keywords indexed in databases: CAPES periodical (SciELO, LILACS, BEDENF) and on the website of the Brazilian Society of Stomatherapy (SOBEST). The analysis of the articles, emerged two categories: forms of care carried out by the person with the varicose ulcer and the lifestyle of the person with varicose ulcer. During the research it was found that most of the studies focused on the process of development and healing of the lesions. In addition, it was possible to observe the importance of knowing the style of life of the person with varicose ulcer, since this can interfere or not in the care process.

Keywords: care, health, nursing, varicose ulcer.

\section{RESUMEN}

El cliente con úlcera venosa requiere el cuidado apropiado, con miras la restauración de la salud y retomar las actividades cotidianas. El objetivo de este estudio consiste en identificar los hábitos de vida de la persona con úlcera varicosa y caracterizar las formas de atención

realizada por la persona con úlcera varicosa. Una búsqueda en línea de artículos publicados en el período comprendido entre 2009 y 2014 que abordó el tema abordado por palabras clave indexadas en las bases de datos: periódico de CAPES (LILACS, SciELO, BEDENF) y de la Sociedad Brasileña de Estoma- 
terapía (SOBEST). Del análisis de los artículos, emergieron dos categorías: formas de cuidado que realizan con la úlcera varicosa y el estilo de vida de la persona con úlcera varicosa. Durante la investigación se observó que la mayoría de los estudios se centra en el proceso de elaboración y curación de las lesiones. Además, fue posible observar la importancia de conocer el estilo de vida de la persona con úlcera varicosa, ya que esto puede interferir o no en el proceso de los cuidados.

Palabras clave: cuidados, salud, enfermería, úlcera varicosa.

\section{RESUMO}

O cliente com úlcera venosa requer cuidados adequados, com vistas de restaurar a saúde e retornar às atividades diárias. Os objetivos deste estudo são: identificar os hábitos de vida da pessoa com úlcera varicosa e caracterizar as formas de cuidados realizadas pela pessoa com úlcera varicosa. Foi realizada uma pesquisa on-line de artigos publicados no período entre 2009 e 2014 que abordou o tema aos foram pesquisados utilizando palavras-chave cadastradas nos bancos de dados: periódico CAPES (SciELO, LILACS, BEDENF) e no site da Sociedade Brasileira de Estomaterapia (SOBEST). Da análise dos artigos, emergiram duas categorias: formas de cuidados realizadas pela pessoa com a úlcera varicosa e o estilo de vida da pessoa com úlcera varicosa. Durante a pesquisa verificou-se que a maioria dos estudos focava o processo de desenvolvimento e cicatrização das lesões. Além disso, foi possível observar a importância de conhecer o estilo de vida da pessoa com úlcera varicosa, já que este pode interferir ou não no processo de cuidados.

Palavras chave: cuidado, saúde, enfermagem, úlcera varicosa.

\section{INTRODUÇÃO}

A pessoa com úlcera varicosa necessitam de cuidados apropriados e de forma resolutiva, com vista ao restabelecimento da saúde e seu retorno às atividades cotidianas (Malaquias, 2012). Pesquisas indicam que os Estados Unidos têm uma prevalência estimada em 500.000 a 800.000 casos, sendo, provavelmente, subestimados devido ao envelhecimento populacional (Costa,2011).

$\mathrm{Na}$ Europa e Austrália, a incidência varia de $0,3 \%$ a $1 \%$ da população total, enquanto que, mundialmente, gira em torno de 2,7\%. Quando as pesquisas abrangem úlceras ativas e cicatrizadas, a prevalência varia de $1 \%$ a $1,3 \%$ (Silva, 2012).

No Brasil, poucos estudos indicam a porcentagem da ocorrência de tratamento de pessoas com úlceras venosas nos ambulatórios unidades básicas de saúde e em hospitais através de registros em fichas cadastrais e/ou prontuários, principalmente o número de pessoas com feridas cicatrizadas após o uso de alguma terapia medicamentosa ou não (Sant'Anna, 2012).

Além disso, pesquisas apontam que aproximadamente $3 \%$ da população nacional possuem um tipo de lesão, aumentando para 10\% em pessoas com diabetes, somando um total de valores próximos a quatro milhões de indivíduos que estejam acometidas por lesões crônicas ou tenham algum tipo de complicação no processo de cicatrização (Santos,2011)

Justifica-se a realização deste estudo a importância de conhecer as formas de cuidado realizada a pessoa com úlcera varicosa, considerando a importância de um atendimento adequado a esta população no que diz respeito avaliação das lesões, realização de curativos e encaminhamentos necessários, além de ações educativas para evolução favorável ao processo 
de cicatrização e prevenção do aparecimento de lesões e ocorrência de recidivas.

Este estudo tem como objetivos identificar o estilo de vida da pessoa com úlcera varicosa e caracterizar as formas de cuidado realizadas à pessoa com úlcera varicosa.

\section{REFERENCIAL CONCEITUAL}

\section{Cuidado}

O cuidado com a vida é uma arte que antecede todas as outras, sem a qual não seria possível a existência. Está na origem de todos os conhecimentos e na matriz de todas as criaturas. Embora inserida na vida de todos, ainda permanece nos dias atuais, tão desconhecida como o seu manejo (Waldow, 2001).

Boff (2003) em Saber cuidar: ética do humano compaixão pela terra, trás de maneira simples e clara o significado do que é o cuidado:

[...] O cuidado transcende o próprio ato $e$ passa a ser uma atitude que abrange um momento de atenção, de zelo, e de desvelo. É uma ocupação, preocupação, responsabilização e envolvimento afetivo com o outro [...].

Dispor de um tempo para cuidar do outro é disponibilizar todos seus conhecimentos, habilidades, emoções, transmitindo afeto e sensibilidade. $\mathrm{O}$ cuidado em enfermagem abarca as competências e habilidades técnicas e científicas, bem como as dimensões relacionais.

Durante a pesquisa, foi observado que o manejo do cuidado está focado ao tratamento da ferida quanto à realização do curativo e a efetividade dos produtos utilizados, com caráter significativo às práticas higiênicas (limpeza com soro fisiológico) e o tipo de coberturas específicas, como hidrocolóide, hidrogel, AGE, entre outros (Martins, 2008).

\section{Úlcera Varicosa}

Feridas crônicas podem ser definidas como aquelas que não cicatrizaram espontaneamente em três meses e que, frequentemente, apresentam como complicação processos infecciosos, podendo ser consideradas feridas complexas, sobretudo quando associadas com patologias sistêmicas que prejudiquem o processo de cicatrização (Fonseca, 2012).

A úlcera varicosa faz parte do diagnóstico diferencial das úlceras crônicas dos membros inferiores, assim consideradas quando não cicatrizam dentro do período de seis semanas. As demais causas de úlceras crônicas nos membros inferiores são: a insuficiência arterial, a neuropatia, o linfedema, a artrite reumatoide, os traumas, a osteomielite crônica, a anemia falciforme, as vasculites, os tumores cutâneos (Tavares, 2012).

Recentemente, dados retirados do Vigitel (2013), demonstraram que $12,9 \%$ das mulheres são tabagistas, enquanto que $15,5 \%$ dos homens possuem esse hábito, indicando assim a importância de consultas regulares focadas na prevenção, já que o tabagismo é um fator que contribui negativamente no processo de cicatrização de feridas (Costa, 2011).

Independente da faixa etária acometida, observamos impactos tanto no aspecto físico como psicossocial, como a dor, dificuldades para locomoção, limitações no trabalho doméstico, impedimento de realizar atividades sociais, vergonha de expor as pernas, limitação na prática de esportes, lazer e restrições na vida conjugal, necessitando assim de cuidados apropriados e de forma resolutiva, com vista ao restabelecimento da saúde e seu retorno aos afazeres do cotidiano (Malaquias, 2012).

Considerando a importância de um atendimento adequado a esta população, há necessidade da atuação de uma equipe multiprofissional, na qual está inserida a Enfermagem, que se destaca por prestar atendimento, na 
avaliação ampliada das pessoas com úlcera venosa, avaliação das lesões, realização de curativos e encaminhamentos necessários, além de ações educativas para evolução favorável do processo de cicatrização e prevenção do aparecimento de lesões e ocorrência de recidivas (Dantas, 2010).

\section{Estilo de vida}

O desenvolvimento de ações direcionadas a redução dos fatores de risco modificáveis no âmbito da atenção primária demanda a presença de profissionais que detenham o domínio de métodos adequados para abordar os problemas de saúde e dialogar sobre as mudanças de estilo de vida de maneira a reconhecer a subjetividade e as necessidades dos usuários e promovendo assim sua autonomia (Figueira, 2015).

O estilo de vida pode ser definido como um conjunto de decisões individuais que afetam a saúde do indivíduo, as quais poderão exercer certo grau de controle frente às decisões e os hábitos pessoais para a saúde (Lalonde, 1974). Por ser uma atitude adotada pelo indivíduo que julga como correta para a manutenção de sua saúde, este, em algum momento, pode ser considerado responsável pelo desenvolvimento de doenças as quais poderão modificar o cotidiano e a realização de suas atividades habituais.

Mudar o estilo de vida não é uma tarefa fácil, geralmente vem acompanhada pela resistência. Por isso a maioria das pessoas não acolhe as modificações necessárias dos hábitos alimentares, na realização de prática de exercícios físicos e, especialmente a manutenção dessas mudanças. Assim, a educação em saúde aparece como uma alternativa fundamental para conduzir as pessoas a essas transformações, para fins de prevenção e/ou controle dos fatores de risco (Santos, 2008).
O trabalho educativo em grupos consiste assim em uma das alternativas para se buscar a promoção da saúde que permite o aprofundamento de discussões e a ampliação de conhecimentos, de modo que as pessoas superem suas dificuldades, co-responsabilizando-se pelo cuidado de sua saúde, obtendo assim maior autonomia, melhores condições e qualidade de vida (Silva, 2012).

\section{METODOLOGIA}

Trata-se de uma pesquisa realizada por meio de uma revisão integrativa, a qual possibilita divulgar uma amplitude de conhecimentos, pois um único estudo proporciona ao leitor os resultados de várias pesquisas ( $\mathrm{Fi}-$ gueiredo, 2004)

A revisão integrativa de literatura é um método específico, que resume o passado da literatura empírica ou teórica, para fornecer uma compreensão mais abrangente de um fenômeno particular, pois esse método de pesquisa objetiva traçar uma análise sobre $o$ conhecimento já construído em pesquisas anteriores sobre um determinado tema. A revisão integrativa possibilita a síntese de vários estudos já publicados, permitindo a geração de novos conhecimentos, pautados nos resultados apresentados pelas pesquisas anteriores (Mendes, 2008).

A coleta de dados ocorreu em janeiro e fevereiro de 2014. Foram acessados artigos publicados no período de 2009 a 2014 que se aproximavam com a temática abordada, indexados na base de dados no periódico CAPES que inclui SciELO (Scientific Eletronic Library Online), LILACS, BIREME, MEDLINE. Além disso, foi realizada uma busca no site da SOBEST (Sociedade Brasileira de Estomaterapia) por meio das seguintes descritores: cuidado, gênero e úlceras varicosas separadas pelo ope- 
rador and. Na base internacional (MEDLINE) foi utilizado somente à pesquisa referente à úlcera varicosa com o descritor varicose ulcer. Tais bases apresentam fontes fidedignas para pesquisa, pois se trata de uma biblioteca eletrônica amparada pelo Centro Latino-Americano e do Caribe de Informação em Ciências da Saúde (Bireme) com inúmeras coleções de periódicos brasileiros disponíveis em textos completos on-line.

Como critério de inclusão, foi utilizado artigos na íntegra publicados no período de 5 anos (2009 à 2014). E, como critério de exclusão, os artigos que não se aproximavam do tema proposto, os que se apresentavam somente em forma de resumo. Além disso, dissertações e teses científicas não foram contemplados neste estudo.

Mesmos se tratando de uma pesquisa, não foi necessário que esse estudo submetesse à aprovação do Comitê de Ética em Pesquisa, uma vez que manuseia dados de livre acesso, não se tratando, portanto, de documentos que demandem sigilo ético.

\section{RESULTADOS}

Durante as buscas, 68 artigos foram apresentados com a inserção do descritor úlcera varicosa, sendo utilizado apenas 10 artigos. No decorrer das pesquisas utilizando o descritor cuidado, 931 artigos foram encontrados. Destes, 17 artigos apresentavam aproximação com a temática a ser discutida. Ao inserir os descritores úlcera varicosa and cuidado, foram encontrados 96 artigos, sendo utilizados no estudo somente 23 artigos, os quais se aproximavam do tema proposto.

A avaliação dos dados permitiu visualizar que os estudos com abordagem sobre o cuidado são importantes para o campo da enfermagem, pois as mudanças sociais ao longo das décadas e o perfil de patologias de curso crônico abrem um espaço no campo científico sobre as formas com que indivíduos. Os conteúdos dos estudos foram organizados em duas categorias: formas de cuidado realizadas em úlcera varicosa e os hábitos de vida da pessoa com úlcera varicosa.

\begin{tabular}{|lcc|}
\hline \multicolumn{3}{|c|}{ QUADRO I: ARTIGOS PESQUISADOS 2014 } \\
DESCRITORES & TOTAL & ARTIGOS CITADOS \\
& ARTIGOS & $\begin{array}{c}\text { QUE SE APROXIMAM } \\
\text { DO TEMA }\end{array}$ \\
CUIDADO & 931 & 17 \\
ÚlLERA VARICOSA & 68 & 10 \\
ÚLCERA VARICOSA & & 06 \\
AND CUIDADO & 10 & 06 \\
\hline
\end{tabular}

\section{DISCUSSÃO}

\section{Formas de cuidado realizadas em úlcera va- ricosa.}

Durante a pesquisa, foram encontrados artigos que apontavam formas e características das úlceras varicosas, dimensão, coloração, localização, tempo do desenvolvimento da ferida e de cicatrização com referências aos produtos utilizados para cuidado com a lesão de perna. Contudo, esses dados são apresentados de forma estimada, já que pouco se registra a evolução do processo cicatricial de maneira sistemática. Além disso, pouco se refere ao contexto sociodemográfico e cultural de pessoas com úlcera varicosa. Não há estudos que abordem essa questão de maneira descritiva, com aprofundamento sobre a temática. A maioria dos estudos apontam estas problemáticas de forma pontual e/ou superficial.

Durante a busca, foram evidenciadas diversas formas de tratamento para úlceras varicosas. Dentre as opções para realização de 
curativos em úlcera varicosa, a terapia mais utilizada é o AGE (Ácidos Graxos Essenciais) ou qual possui poder hidratante e fator de proteção em pele íntegra e cicatrizante em pele lesionada. Este produto contém Vitaminas A e E, Lecitina de Soja que agem promovendo hidratação, além de possuírem propriedades emolientes que protegem a pele, auxiliando no processo de cicatrização de feridas (Silva, 2012).

O cuidado clínico de enfermagem a pessoa com de úlcera varicosa permeia vários aspectos. O profissional realiza a coleta de um breve histórico do paciente, detendo-se nos aspectos relacionados aos membros inferiores, realiza anamnese e exame físico. Após detectar os possíveis problemas, traça-se um plano de intervenções e posteriormente se analisam os resultados de suas ações, objetivando a priori a cicatrização efetiva da lesão, prevenção de possíveis complicações, orientação para o autocuidado e redução das recidivas (Silva, 2009).

Ao tomarmos a decisão de realizar o ato de cuidar, é preciso valorizar o indivíduo e sua historia, aos quais precisam ser respeitados e inseridos no contexto desse cuidado, permitindo a realização de uma assistência eficaz, individual e holística.

\section{Estilo de vida da pessoa com úlceras varico-} sas.

A pesar de ser um tema de grande relevância e de ocorrência constante, ainda nos dias atuais o número de pessoas com doenças crônicas, em destaque às úlceras varicosas, estão presentes em todos os estados do Brasil, porém os registros para uma contagem fidedigna ainda encontra-se de maneira insipiente.

Independente da faixa etária acometida, foi observado impacto tanto no aspecto físico como psicossocial, que incluem a dor, di- ficuldades para se locomover, limitações nos afazeres domésticos, nas atividades sociais, vergonha de expor as pernas, limitação nos momentos de lazer e restrições na vida conjugal. Situações estas que podem interferir nas relações para conquista de um emprego, levando a exclusão deste indivíduo do mercado de trabalho (Malaquias, 2012).

Pesquisas realizadas no Brasil indicam que a faixa etária predominante da população afetada é de 51-70 anos, enquanto que estudos realizados em países da Europa indicam faixa etária predominante de 70 anos ou mais (Silva, 2012)

Os estudos a respeito da prevalência e incidência de úlcera varicosa ainda são tímidos e pouco se conhece sobre sua distribuição no Brasil e nas diferentes regiões do país, constituindo-se em um problema que merece atenção especial por parte dos profissionais da área da saúde (Sant'Anna, 2011).

Diante disso, a Enfermagem possui papel fundamental no atendimento a essa população, uma vez que realiza uma avaliação ampliada e sistemática da pessoa com úlcera varicosa, na realização de curativos e encaminhamentos necessários, além de ações educativas para evolução favorável do processo de cicatrização e a prevenção do aparecimento de lesões evitando assim a ocorrência de recidiva (Silva, 2012).

A prática regular de atividade física reduz o risco de mortes prematuras, doenças cardíacas, cânceres e diabetes tipo II, além de prevenir ou reduzir a hipertensão arterial, a obesidade e a osteoporose (Feitosa, 2011) e a possibilidade de manutenção da saúde, redução de peso, alívio do estresse, fonte de lazer e de qualidade de vida (Callegaro, 2012).

A mudança do estilo de vida enfatizada pelos profissionais de saúde, em destaque o en- 
fermeiro, torna-se uma medida de fundamental importância para o sucesso da terapêutica da pessoa com úlcera venosa. A não adesão ao tratamento está relacionada com presença de dor, o desconforto, a desmotivação, a falta de apoio e o isolamento social (Fonseca, 2012).

Tomar como ponto de partida o conhecimento das condições de vida destes indivíduos que recebem cuidados especializados se faz necessário, pois a compreensão de questões que permeiam o contexto social inserido permite a visualização das causas e os motivos que levaram o desenvolvimento desta ferida. Ter ciência dos problemas sociais envolvidos, permite que o profissional de saúde promova uma assistência individualizada, focal, auxiliando portanto na eficácia do tratamento com acompanhamento contínuo e integral, colaborando assim com o processo de cicatrização, processo este primordial para o sucesso do tratamento.

\section{CONCLUSÃO}

É notória a importância de estudos com enfoque nos cuidados de feridas crônicas, em especial as úlceras varicosas na perspectiva do indivíduo com tal enfermidade, já que, ao interferir no cotidiano, modificando sua rotina, seus afazeres, ela transforma a estrutura natural do corpo.

A alteração da imagem e funcionalidade corporal pode gerar, angústia, dor, sofrimento e impossibilidade de realizar suas atividades cotidianas, além de comprometer a autonomia do sujeito. Esse cuidado implica em ajuda de outra pessoa, quer seja um profissional, quer seja um familiar, cuidador.

$\mathrm{O}$ cuidado realizado pela enfermagem não deve ser focado somente na úlcera em si, pois o seu desenvolvimento pode ser um obstáculo para novas conquistas, sendo um impedimento para sociabilização, levando-o ao isolamen-

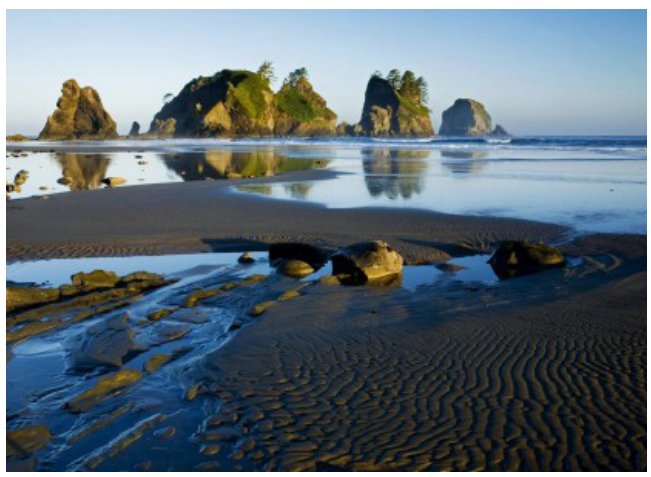

to, a solidão. Conhecer o indivíduo dentro do seu contexto social, cultural e as estratégias utilizadas para o cuidado de feridas, são condições essenciais para o desenvolvimento de atividades com enfoque nas ações de prevenção e promoção à saúde com enfoque no indivíduo com úlcera varicosa. Além disso, valorizar seus conhecimentos e suas práticas diárias, são fatores importantes para estabelecer confiança entre profissionais de saúde e o usuário, pois o trabalho em conjunto associando o saber popular com os conhecimentos científicos, estimula a corresponsabilização dinamizando o processo do cuidado, contribuindo para compreensão quanto importância da mudança dos hábitos de vida quando necessário.

\section{REFERÊNCIAS}

- Boff, L. (2003). Saber cuidar: ética do humano compaixão pela terra. Petrópolis: Vozes..

- Collière, M.F. (2003). Cuidar ... a primeira arte da vida. Lisboa: Lusodidacta.

- Costa, I. K. F. (2011). Pessoas com úlceras venosas: estudo do modo psicossocial do modelo adaptativo de Roy. Rev. Gaúcha Enferm, 32(3), 561-568.

- Dantas, D.V., Torres, G.V. (2010). Assistência a portadores de ulceras venosas baseada em protocolos: revisão de literatura em bases de dados eletrônicas. Rev enferm UFPE, 4 (6), 1944-1950.

- Ferreira, A. M., et al (2012). Utilização dos ácidos graxos 
no tratamento de feridas: uma revisão integrativa da literatura nacional. Rev Esc Enferm USP, 46(3), 752-760.

- Fonseca, C., Franco, T., Ramos, A., \& Silva, C. (2012). A pessoa com úlcera de perna, intervenção estruturada dos cuidados de enfermagem: revisão sistemática da literatura. Rev Esc Enferm USP , 46(2), 480-486.

- Malaquias, S. G; et al (2012). Pessoas com úlceras vasculogênicas em atendimento ambulatorial de enfermagem: estudo das variáveis clínicas e sociodemográficas. Rev Esc Enferm USP, 46(2), 302-310.

- Martins, M. A (2008). Avaliação de feridas crônicas de pacientes ambulatoriais de Unidades Básicas de Saúde, em Goiânia/GO (dissertação). Universidade Federal de Goiás: Brasil.

- Mendes, K.D.S., Silveira, R.C.C.P., Galvão, C.M (2008). Revisão integrativa: método de pesquisa para a incorporação de evidências na saúde e na enfermagem. Texto Contexto Enferm, 17(4), 758-764.

- Sant’Anna, S. M. S.C. et al. (2012). Úlceras venosas: caracterização clínica e tratamento em usuários atendidos em rede ambulatorial. Rev Bras Enferm 65(4), 637-644.

- Santos, J.S., Vieira, A. B. D., \& Kamada, I. (2009). A Rosa Mosqueta no tratamento de feridas abertas: uma revisão. Rev Bras Enferm, 62(3), 457-462.

- Silva, F. A. A. et al (2015). Enfermagem em estomaterapia: cuidados clínicos ao portador de úlcera venosa. Rev Bras Enferm, 62 (6), 889-893.

- Silva, M.A.M. et al. (2012). Resultados do tratamento da Insuficiência Venosa Crônica grave com espuma de polidocanol guiada por ultrassom. J Vasc Bra, 111(3), 206-211.

- Silva, P. A. S.et al (2012). A saúde do homem na visão dos enfermeiros de uma unidade básica de saúde. Escola Anna Nery, 16(3) 561-568.

- Tavares, E et al. (2011). Úlcera de Marjolin associada a ulceração e osteomielite crônicas. An Bras Dermatol, 86(2), 366-369.

- Waldow, V. R (2001). Cuidado Humano. O resgate necessário. Porto Alegre: Sagra Luzzatto. 